\title{
Editorial \\ Publisher's Note: We Changed Page Numbers to Article Numbers for Articles Published in Journal of Xenobiotics Volumes 1-9
}

\author{
Journal of Xenobiotics Editorial Office
}

MDPI AG, St. Alban-Anlage 66, 4052 Basel, Switzerland; jox@mdpi.com

check for updates

Citation: Journal of Xenobiotics Editorial Office. Publisher's Note: We Changed Page Numbers to Article Numbers for Articles Published in Journal of Xenobiotics Volumes 1-9. J. Xenobiot. 2022, 12, 53-55. https:// doi.org/10.3390/jox12010005

Received: 1 March 2022 Accepted: 2 March 2022 Published: 3 March 2022

Publisher's Note: MDPI stays neutral with regard to jurisdictional claims in published maps and institutional affiliations.

Copyright: (C) 2022 by the author. Licensee MDPI, Basel, Switzerland. This article is an open access article distributed under the terms and conditions of the Creative Commons Attribution (CC BY) license (https:// creativecommons.org/licenses/by/ $4.0 /)$.
The Journal of Xenobiotics [1] was published by PAGEPress from Volume 1 (2011) to Volume 9 (2019). Since Volume 10, Issue 1 (2020), the Journal of Xenobiotics has been published by MDPI.

Previous articles in Volumes 1-9, published by PAGEPress in open access under a CC-BY (or CC-BY-NC-ND) license, are now hosted by MDPI on mdpi.com as a courtesy and upon agreement with PAGEPress.

To standardize the metadata format of all previous articles, MDPI republished 92 articles in Volumes 1-9 with article numbers replacing page numbers (Table 1). MDPI also corrected Issue Number errors that occurred during importing of batch data for 14 articles in Volume 3 (Table 2).

Table 1. MDPI changed page numbers to articles numbers for 92 articles in Volumes 1-9.

\begin{tabular}{|c|c|c|}
\hline DOI & Previous Page Number & Current Article Number \\
\hline 10.4081/xeno.2011.e1 & $1-5$ & e1 \\
\hline 10.4081/xeno.2011.e2 & $6-8$ & e2 \\
\hline 10.4081/xeno.2011.e3 & $9-16$ & e3 \\
\hline 10.4081/xeno.2011.e4 & $17-21$ & e4 \\
\hline 10.4081/xeno.2011.e6 & $29-34$ & e6 \\
\hline 10.4081/xeno.2011.e5 & $22-28$ & e5 \\
\hline 10.4081/xeno.2011.e7 & $35-37$ & $\mathrm{e} 7$ \\
\hline 10.4081/xeno.2011.e8 & $38-44$ & e8 \\
\hline 10.4081/xeno.2012.e1 & $1-6$ & e1 \\
\hline 10.4081/xeno.2012.e2 & $7-12$ & $\mathrm{e} 2$ \\
\hline 10.4081/xeno.2012.e3 & $13-17$ & e3 \\
\hline 10.4081/xeno.2012.e4 & $18-23$ & e4 \\
\hline 10.4081/xeno.2012.e5 & $24-27$ & e5 \\
\hline 10.4081/xeno.2012.e6 & $28-34$ & e6 \\
\hline 10.4081/xeno.2012.e7 & $35-40$ & e7 \\
\hline 10.4081/xeno.2012.e8 & $41-42$ & e8 \\
\hline 10.4081/xeno.2012.e9 & $43-46$ & e9 \\
\hline 10.4081/xeno.2012.e10 & $47-52$ & e10 \\
\hline 10.4081/xeno.2013.e1 & $1-8$ & $\mathrm{e} 1$ \\
\hline 10.4081/xeno.2013.e2 & $9-13$ & $\mathrm{e} 2$ \\
\hline 10.4081/xeno.2013.e3 & $14-23$ & e3 \\
\hline 10.4081/xeno.2013.e4 & $19-23$ & $\mathrm{e} 4$ \\
\hline $10.4081 /$ xeno.2013.e5 & $24-28$ & e5 \\
\hline 10.4081/xeno.2013.e6 & $29-35$ & e6 \\
\hline 10.4081/xeno.2013.e7 & $36-39$ & e7 \\
\hline 10.4081/xeno.2013.e8 & $40-48$ & e8 \\
\hline 10.4081/xeno.2013.s1.e1 & $1-2$ & e1 \\
\hline 10.4081/xeno.2013.s1.e2 & $3-5$ & e2 \\
\hline 10.4081/xeno.2013.s1.e3 & $6-7$ & e3 \\
\hline
\end{tabular}


Table 1. Cont.

\begin{tabular}{|c|c|c|}
\hline DOI & Previous Page Number & Current Article Number \\
\hline 10.4081/xeno.2013.s1.e4 & $8-10$ & e4 \\
\hline 10.4081/xeno.2013.s1.e5 & $11-13$ & e5 \\
\hline 10.4081/xeno.2013.s1.e6 & $14-16$ & e6 \\
\hline 10.4081/xeno.2013.s1.e7 & $17-19$ & e7 \\
\hline 10.4081/xeno.2013.s1.e8 & $20-22$ & e8 \\
\hline 10.4081/xeno.2013.s1.e9 & $23-25$ & e9 \\
\hline 10.4081/xeno.2013.s1.e10 & $26-28$ & e10 \\
\hline 10.4081/xeno.2013.s1.e11 & $29-30$ & e11 \\
\hline 10.4081/xeno.2013.s1.e12 & $31-33$ & $\mathrm{e} 12$ \\
\hline 10.4081/xeno.2013.s1.e13 & $34-35$ & e13 \\
\hline 10.4081/xeno.2013.s1.e14 & $36-39$ & e14 \\
\hline 10.4081/xeno.2014.1991 & $1-7$ & 1991 \\
\hline 10.4081 /xeno. 2014.2240 & $8-13$ & 2240 \\
\hline 10.4081/xeno.2014.2264 & $20-28$ & 2264 \\
\hline 10.4081/xeno.2014.2272 & 14-19 & 2272 \\
\hline 10.4081/xeno.2014.3945 & 29-39 & 3945 \\
\hline 10.4081/xeno.2014.4596 & $40-45$ & 4596 \\
\hline 10.4081/xeno.2014.4680 & $46-52$ & 4680 \\
\hline 10.4081/xeno.2014.4891 & $53-55$ & 4891 \\
\hline 10.4081/xeno.2014.4892 & $56-58$ & 4892 \\
\hline 10.4081/xeno.2014.4893 & $59-61$ & 4893 \\
\hline 10.4081/xeno.2014.4894 & $62-64$ & 4894 \\
\hline 10.4081/xeno.2014.4895 & $65-67$ & 4895 \\
\hline 10.4081/xeno.2014.4896 & $68-69$ & 4896 \\
\hline 10.4081/xeno.2014.4897 & $70-72$ & 4897 \\
\hline 10.4081/xeno.2014.4898 & $73-75$ & 4898 \\
\hline 10.4081 /xeno.2014.4899 & $76-77$ & 4899 \\
\hline 10.4081/xeno.2014.4900 & $78-80$ & 4900 \\
\hline 10.4081 /xeno.2014.4901 & $81-84$ & 4901 \\
\hline 10.4081/xeno.2014.4902 & $85-87$ & 4902 \\
\hline 10.4081/xeno.2014.4903 & $88-91$ & 4903 \\
\hline 10.4081/xeno.2014.4904 & $92-93$ & 4904 \\
\hline 10.4081/xeno.2014.4905 & $94-96$ & 4905 \\
\hline 10.4081/xeno.2015.5086 & $8-14$ & 5086 \\
\hline 10.4081/xeno.2015.5125 & $1-7$ & 5125 \\
\hline 10.4081/xeno.2015.5264 & 15-19 & 5264 \\
\hline 10.4081/xeno.2015.5369 & $20-23$ & 5369 \\
\hline $10.4081 /$ xeno.2015.5769 & $27-30$ & 5769 \\
\hline 10.4081/xeno.2015.5770 & $31-33$ & 5770 \\
\hline 10.4081/xeno.2015.5771 & $24-26$ & 5771 \\
\hline 10.4081/xeno.2015.5772 & $34-36$ & 5772 \\
\hline 10.4081/xeno.2015.5773 & $37-39$ & 5773 \\
\hline 10.4081/xeno.2015.5781 & $40-41$ & 5781 \\
\hline 10.4081/xeno.2016.5660 & $19-24$ & 5660 \\
\hline 10.4081 /xeno.2016.5774 & $1-7$ & 5774 \\
\hline 10.4081/xeno.2016.5889 & $8-13$ & 5889 \\
\hline 10.4081/xeno.2016.6092 & 14-18 & 6092 \\
\hline 10.4081/xeno.2016.6585 & $25-27$ & 6585 \\
\hline 10.4081/xeno.2016.6586 & $28-32$ & 6586 \\
\hline 10.4081/xeno.2016.6587 & $33-35$ & 6587 \\
\hline 10.4081/xeno.2016.6588 & $36-38$ & 6588 \\
\hline 10.4081/xeno.2016.6589 & $39-40$ & 6589 \\
\hline 10.4081/xeno.2016.6721 & $41-44$ & 6721 \\
\hline $10.4081 /$ xeno.2017.6475 & $1-9$ & 6475 \\
\hline 10.4081/xeno.2017.6702 & $10-14$ & 6702 \\
\hline 10.4081/xeno.2017.7101 & $15-20$ & 7101 \\
\hline 10.4081/xeno.2017.7149 & $25-30$ & 7149 \\
\hline
\end{tabular}


Table 1. Cont.

\begin{tabular}{ccc}
\hline DOI & Previous Page Number & Current Article Number \\
\hline 10.4081 /xeno.2017.7173 & $21-24$ & 7173 \\
$10.4081 /$ xeno.2018.7674 & $1-3$ & 7674 \\
10.4081 xeno.2018.7809 & $4-6$ & 7809 \\
10.4081 xeno.2018.7810 & $10-12$ & 7810 \\
10.4081 xeno.2018.7820 & $7-9$ & 7820 \\
10.4081 xeno.2019.8147 & $1-3$ & 8147 \\
\hline
\end{tabular}

Table 2. MDPI's corrected Issue Numbers for 14 articles in Volume 3.

\begin{tabular}{|c|c|c|}
\hline DOI & Previous Issue Number & Current Issue Number \\
\hline 10.4081/xeno.2013.s1.e1 & 11 & s1 \\
\hline 10.4081/xeno.2013.s1.e2 & 11 & s1 \\
\hline 10.4081/xeno.2013.s1.e3 & 11 & s1 \\
\hline 10.4081/xeno.2013.s1.e4 & 11 & s1 \\
\hline 10.4081/xeno.2013.s1.e5 & 11 & s1 \\
\hline 10.4081/xeno.2013.s1.e6 & 11 & s1 \\
\hline 10.4081/xeno.2013.s1.e7 & 11 & s1 \\
\hline 10.4081/xeno.2013.s1.e8 & 11 & s1 \\
\hline 10.4081/xeno.2013.s1.e9 & 11 & s1 \\
\hline 10.4081/xeno.2013.s1.e10 & 11 & s1 \\
\hline 10.4081/xeno.2013.s1.e11 & 11 & s1 \\
\hline 10.4081/xeno.2013.s1.e12 & 11 & s1 \\
\hline 10.4081/xeno.2013.s1.e13 & 11 & s1 \\
\hline 10.4081/xeno.2013.s1.e14 & 11 & s1 \\
\hline
\end{tabular}

Conflicts of Interest: The author declares no conflict of interest.

\section{Reference}

1. Journal of Xenobiotics Homepage. Available online: https://www.mdpi.com/journal/jox (accessed on 10 February 2022). 